\title{
Combining Dissipative Particle Dynamics and Monte Carlo Techniques
}

\author{
S. M. Willemsen, ${ }^{1}$ T. J. H. Vlugt, H. C. J. Hoefsloot, and B. Smit \\ Department of Chemical Engineering, University of Amsterdam, Nieuwe Achtergracht 166, \\ 1018 WV Amsterdam, The Netherlands \\ E-mail: sanderw@chemeng.chem.uva.nl,tampert@ct-cr6.chem.uva.nl, \\ huubh@chemeng.chem.uva.nl,smit@chemeng.chem.uva.nl
}

Received April 28, 1998; revised September 9, 1998

\begin{abstract}
Dissipative particle dynamics (DPD) has been introduced as a method for simulating complex fluids at hydrodynamic time scales. In this work we investigate the possibilities to combine this DPD method with advanced Monte Carlo techniques. We show that this combined approach results in a more efficient sampling scheme to compute thermodynamic properties. We illustrate these advantages by computing a phase diagram of two liquids that de-mix, and the calculation of the chemical potential of a polymer in solution. (c) 1998 Academic Press

Key Words: dissipative particle dynamics; Monte Carlo techniques.
\end{abstract}

\section{INTRODUCTION}

Simulating the hydrodynamics of complex fluids is very challenging because of the wide variety of time scales in these systems. Solving them through standard molecular dynamics (MD) is computationally very expensive, if not impossible. Therefore other methods have been introduced, of which dissipative particle dynamics (DPD) [1] is one. The idea of this method is to simulate hydrodynamic behavior through the motions of soft spheres (fluid particles). It was developed as a combination of several methods; MD, Brownian dynamics, and lattice gas simulations. The commonly used steep MD potential (Lennard-Jones type) is replaced in DPD by a very soft repulsive potential, making larger time steps possible. The lattice gas approach gives the distinction between a collision and propagation phase, but in DPD the motions are not restricted to a lattice (so that the problem of Galilean invariance does not arise). And finally a random force is introduced similar to Brownian dynamics.

Hoogerbrugge and Koelman [1] demonstrated that their original DPD scheme obeys the Navier-Stokes equations. This was eluded even more by Español [2]. Español and

\footnotetext{
${ }^{1}$ Corresponding author.
} 
Warren [3] showed that with a slight modification of the original algorithm a proper thermal equilibrium is found and a Hamiltonian can be defined. Pagonabarraga et al. [4] have shown that it is essential to use a time-reversible scheme to solve the equations of motion, to recover the correct dynamics regardless of the time step chosen.

So far, DPD has been used to simulate a variety of problems. For example, hard-sphere suspensions [5], dilute polymer solutions [6], or phase separation [7]. It is interesting to note that all these applications use the standard DPD algorithm. The fact that for the DPD method a Hamiltonian can be defined implies that we can also us Monte Carlo techniques for the same model as is used in a DPD simulation. In this work, we demonstrate that a combined DPD and Monte Carlo approach can result in more efficient sampling schemes. In particular, we illustrate the advantage of this combined approach with the computation of a phase diagram and the free energy of a polymer in solution.

\section{DISSIPATIVE PARTICLE DYNAMICS}

Dissipative particle dynamics is a simulation technique for simulating complex fluids at hydrodynamic time scales. The DPD scheme consists of the calculation of the position and impulses of interacting (fluid) particles over time. The time evolution of these positions $\left(\mathbf{r}_{i}(t)\right)$ and impulses $\left(\mathbf{p}_{i}(t)\right)$ (for simplicity we take the masses of all particles 1$)$ is given by

$$
\frac{d \mathbf{r}_{i}}{d t}=\mathbf{v}_{i}(t), \quad \frac{d \mathbf{v}_{i}}{d t}=\mathbf{f}_{i}(t)
$$

The force acting on the particles is a combination of three parts:

$$
\mathbf{f}_{i}(t)=\sum_{j \neq i}\left(\mathbf{F}_{i j}^{\mathrm{C}}+\mathbf{F}_{i j}^{\mathrm{D}}+\mathbf{F}_{i j}^{\mathrm{R}}\right)
$$

The first part of the force is the conservative part

$$
\mathbf{F}_{i j}^{\mathrm{C}}= \begin{cases}a_{i j}\left(1-\frac{r_{i j}}{r_{\mathrm{c}}}\right) \hat{\mathbf{r}}_{i j}, & \left(r_{i j}<r_{\mathrm{c}}\right), \\ 0, & \left(r_{i j} \geq r_{\mathrm{c}}\right),\end{cases}
$$

where $a_{i j}$ is the maximum repulsion between particle $i$ and $j, \mathbf{r}_{i j}=\mathbf{r}_{i}-\mathbf{r}_{j}, r_{i j}=\left|\mathbf{r}_{i j}\right|$, $\hat{\mathbf{r}}_{i j}=\mathbf{r}_{i j} /\left|\mathbf{r}_{i j}\right|$, and $r_{\mathrm{c}}$ is the cutoff radius. The second and third forces are the dissipative force and the random force:

$$
\begin{aligned}
& \mathbf{F}_{i j}^{\mathrm{D}}=-\gamma \omega^{\mathrm{D}}\left(r_{i j}\right)\left(\hat{\mathbf{r}}_{i j} \cdot \mathbf{v}_{i j}\right) \hat{\mathbf{r}}_{i j}, \\
& \mathbf{F}_{i j}^{\mathrm{R}}=\sigma \omega^{\mathrm{R}}\left(r_{i j}\right) \theta_{i j} \hat{\mathbf{r}}_{i j},
\end{aligned}
$$

in which $\mathbf{v}_{i j}=\mathbf{v}_{i}-\mathbf{v}_{j}, \omega\left(r_{i j}\right)$ is a weight function which tends to zero for $r=r_{\mathrm{c}}$ and $\theta_{i j}$ is a random number with zero mean and unit variance.

Español and Warren [3] showed that the weight functions and the constants in these forces can be chosen arbitrarily, but they should obey

$$
\begin{aligned}
{\left[\omega^{\mathrm{R}}\left(r_{i j}\right)\right]^{2} } & =\omega^{\mathrm{D}}\left(r_{i j}\right), \\
\sigma^{2} & =2 k_{\mathrm{B}} T \gamma,
\end{aligned}
$$


with $k_{\mathrm{B}}$ the Boltzmann constant. If this condition is met a Hamiltonian exists which is given by

$$
H(r, p)=\sum_{i} \frac{p_{i}^{2}}{2 m_{i}}+\mathrm{U}^{\mathrm{C}}(r),
$$

where $\mathrm{U}^{\mathrm{C}}(r)$ is the potential function that gives rise to the conservative forces $\mathbf{F}^{\mathrm{C}}$ (see Eq. (3)).

\section{MONTE CARLO SIMULATIONS}

In the previous section we have outlined the basic equations of the DPD scheme. These equations lead to a Hamiltonian (Eq. (6)). Given the fact that we are simulating a Hamiltonian system, we could use Monte Carlo techniques to simulate this system.

\subsection{Phase Equilibria Calculations}

For many applications it is important to have a detailed knowledge of the phase diagram of a given model. It is in principle straightforward to obtain such information from a DPD simulation by simulating the system at conditions, where phase separation is expected, and observe the formation of these phases [7]. These types of simulations, however, are very time consuming. If the interfacial tension between the two phases is very low it may require very long simulations to compute such a phase diagram of a given model. Such types of problems can be solved if the DPD technique is combined with, for example, the Gibbs ensemble technique to compute phase equilibria.

3.1.1. Gibbs ensemble technique. Panagiotopoulos [8] derived a new scheme to study phase equilibria usually called the Gibbs ensemble technique. The idea behind this scheme is to simulate two boxes which (together) form a NVT ensemble. However, the two boxes can exchange particles and volume. A Monte Carlo scheme is used to ensure that these two boxes are in thermodynamic equilibrium. If one performs a simulation in a two phase region at equilibrium one box will contain one phase while the other box will contain the other phase. Because the two phases are in separate boxes there is no interface in these simulations, and the coexisting density follows directly from the density in the two boxes. In a DPD simulation the formation of this interface makes such a simulation expensive (both in simulation time and number of particles). Details on the Gibbs ensemble simulation are given in the Appendix.

3.1.2. Results. We have computed the phase diagram of the model studied by Groot and Warren [9]. The model of Groot and Warren is an example of liquid-liquid phase separation. Details on the parameters of the model and the simulations are given in the Appendix. The results of the DPD simulations and the Gibbs ensemble simulations are compared in Fig. 1.

As can be seen from the graph, the results of both simulation methods are in good agreement. Such an agreement can be expected from theoretical arguments and, therefore, is a very good test for the methods that these two completely independent techniques give the same results.

It is interesting to compare the efficiency of the two methods. The Gibbs ensemble simulations were performed with $N=1296$ and 384 particles. We did not observe significant 


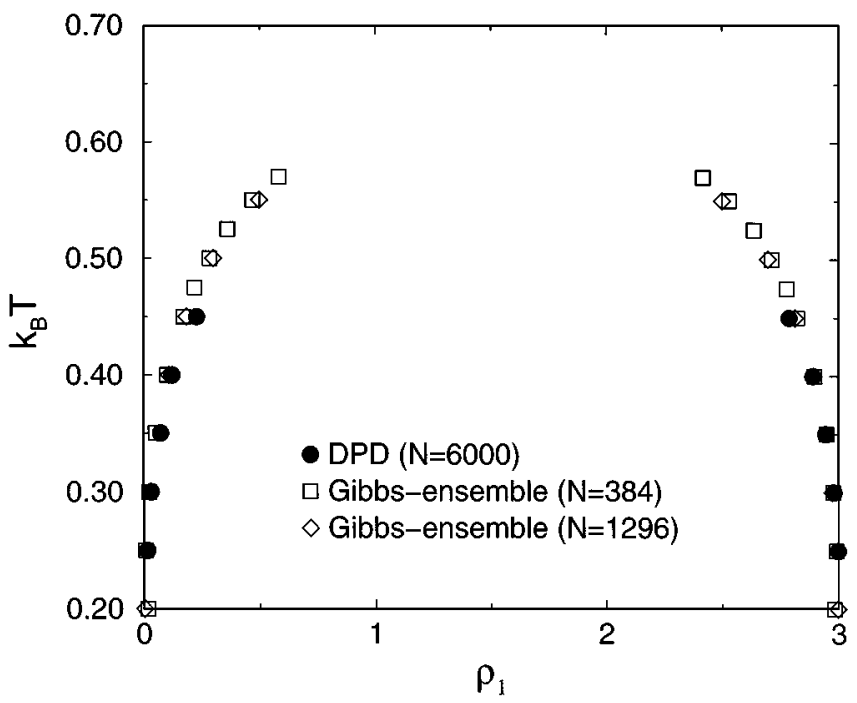

FIG. 1. Phase diagram as calculated with DPD and Gibbs ensemble. $\rho_{1}$ is the concentration of one fluid in the two phases.

finite-size effects, which is in agreement with other studies [10-13]. Therefore, we could obtain the phase diagram shown in Fig. 1 with $N=384$ particles only. The calculation of one coexisting point required approximately $30 \mathrm{~min}$ on an IBM workstation. For the DPD simulations 6000 particles were required and the total simulation time was $4.5 \mathrm{~h}$ on the same computer. Close to the critical point $\left(T_{\mathrm{c}}=0.60\right)$ it was impossible to obtain a stable planar interface for the given system size, with the DPD method because the interfacial tension was too low. With the Gibbs ensemble we could obtain results much closer to the critical point.

From these simulations it is clear that DPD and Gibbs ensemble simulations lead to the same results and both methods can be used, depending on what is the quantity of interest. Obviously the Gibbs ensemble technique is much more efficient for the study of phase equilibria since the equilibrium is reached faster and with less particles.

\section{FREE ENERGY CALCULATIONS}

In applications one would like to map the DPD parameters to real systems. Groot and Warren [9] have shown that for polymers one can use a thermodynamic route and map the DPD parameters on Flory-Huggins parameters. Therefore, it is important to compute thermodynamic quantities of a given DPD model. With standard DPD techniques, however, it is difficult to compute quantities related to the free energy. It would require many simulations to determine the complete equation of state. Also, for this problem the DPD technique can be combined with Monte Carlo techniques to determine the free energy from a single simulation, such as the Widom test particle method [14] or the overlapping distribution method [15].

In this work we are interested in computing the chemical potential of the polymers that are used by Groot and Warren [9]. For chain molecules one can compute the chemical potential using configurational-bias Monte Carlo techniques [16-19]. 


\subsection{Configurational-Bias Monte Carlo}

The configurational-bias Monte Carlo (CBMC) technique is based on an algorithm developed by Rosenbluth and Rosenbluth $[16,19,20]$ for lattice models that has been extended to continuum models [16-19].

We consider a solvent of DPD particles in which we compute the chemical potential of a chain with length $l$. The beads of the chain are connected to each other with a spring which leads to a bond potential given by

$$
U^{\text {bond }}(r)=-\frac{1}{2} C r^{2}+U^{\mathrm{C}}(r),
$$

in which $C$ is the spring constant chosen 2.0 in this study, and $U^{\mathrm{C}}(r)$ is the potential that gives rise to the conservative force $\mathbf{F}^{\mathrm{C}}$ (see Eq. (3)). The method to compute the free energy consists of the following steps:

1. At a random trial position, the first DPD particle of the chain is inserted and the Rosenbluth weight $\left(w_{1}=\exp \left(-\beta u_{1}\right)\right)$ calculated, where $u_{1}$ is the energy of the inserted particle and $\beta=1 / k_{\mathrm{B}} T$.

2. For all next particles $i=2,3, \ldots, l$ in the chain, $k$ positions on a sphere with radius 1 are generated. These vectors are multiplied by a random chain length calculated from the distribution given by $r^{2} \exp \left(-U^{\text {bond }}\right)$. From these $k$ positions we select one, say $n$, with a probability proportional to its Rosenbluth weight:

$$
p_{i}(n)=\frac{\exp \left(-\beta u_{i}(n)\right)}{w_{i}},
$$

where $u_{i}(n)$ is the energy of the $n$th trial position and

$$
w_{i}=\sum_{j=1}^{k} \exp \left(-\beta u_{i}(j)\right),
$$

where $u_{i}(j)$ is the potential energy with the other particles in the system (excluding the bond potential).

3. Step 2 is repeated until the entire chain is grown. The total Rosenbluth factor of the chain is the product of the Rosenbluth factors of each segment divided by the total number of segments:

$$
W=w_{1} \prod_{j=2}^{l} \frac{w_{j}}{k} .
$$

The excess chemical potential is calculated from

$$
\mu_{\mathrm{ex}}=\mu_{\text {test }}-\mu_{0}=-k_{\mathrm{B}} T \ln \langle W\rangle-\left(-k_{B} T \ln \left\langle W^{0}\right\rangle\right),
$$

where $\langle W\rangle$ is the mean Rosenbluth weight (resulting in a chemical potential $\mu_{\text {test }}$ ), and $\left\langle W^{0}\right\rangle$ is the mean Rosenbluth weight of one isolated chain of length $l$ (resulting in a chemical potential $\left.\mu_{0}\right)$. The chemical potential of one isolated chain $\left(\mu_{0}\right)$ has to be calculated from a separate simulation of a chain without the solvent. Since no solvent has to be calculated this is not very time consuming. It is important to note that this chemical potential does not correspond to the ideal gas chemical potential, since in the generation of the trial configurations in the CBMC scheme the bond potential is not considered in the Rosenbluth factor [15]. 


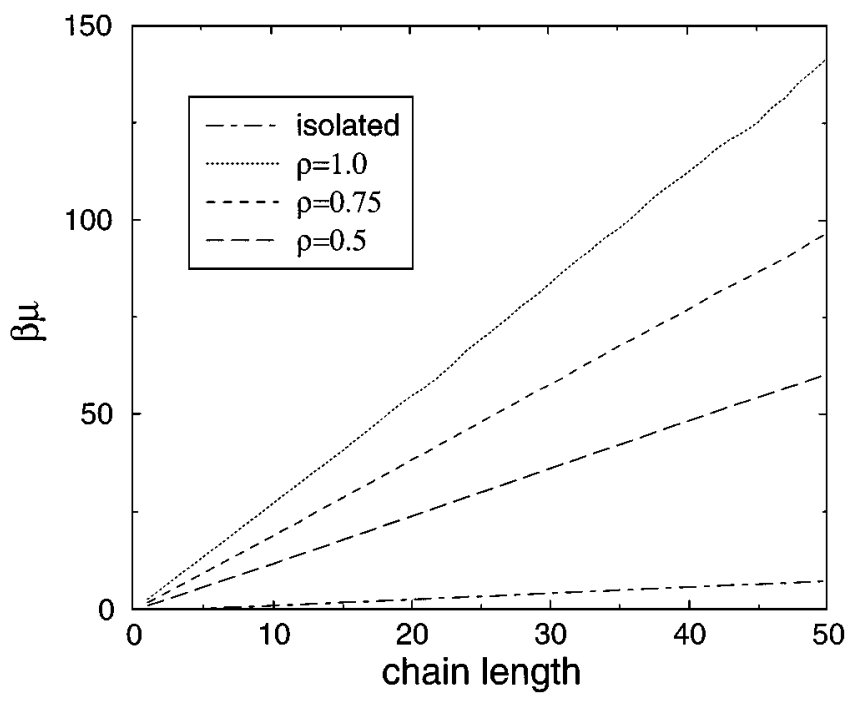

FIG. 2. Chemical potential vs chain length at various densities. For $\rho=0.5,0.75$, and $1.0, \beta \mu_{\text {test }}$ is shown, and for the isolated chain $\beta \mu_{0}$ is shown.

4.1.1. Results. The chemical potential was computed as a function of chain length at various densities. Simulation details are given in the Appendix. The results are shown in Fig. 2. As can be seen the chemical potential grows linear with chain length. To calculate the error in these computations the block average method described by Flyvbjerg and Petersen [21] is used. Results from these computations are depicted in Fig. 3. For low densities the error grows slowly with chain length, but for the higher densities an erratic behavior in the error is observed. This larger error at the highest density might be the explanation of the small wiggles that can be seen in Fig. 2 for $\rho=1.0$. In addition, this erratic behavior may indicate that the sampling scheme is not adequate, as has been observed before [22]. To investigate this further we use the overlapping distribution method.

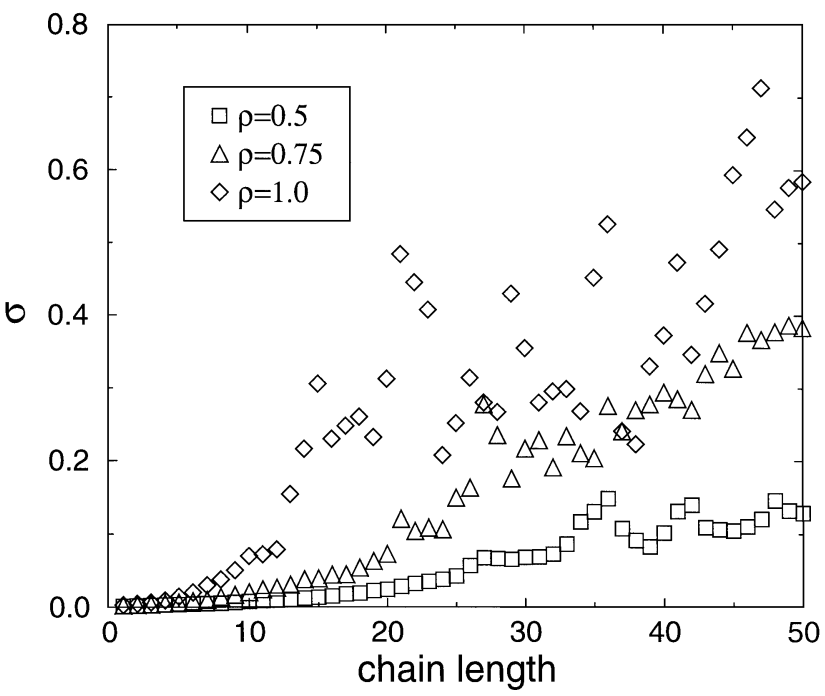

FIG. 3. Error vs chain length at various densities. 


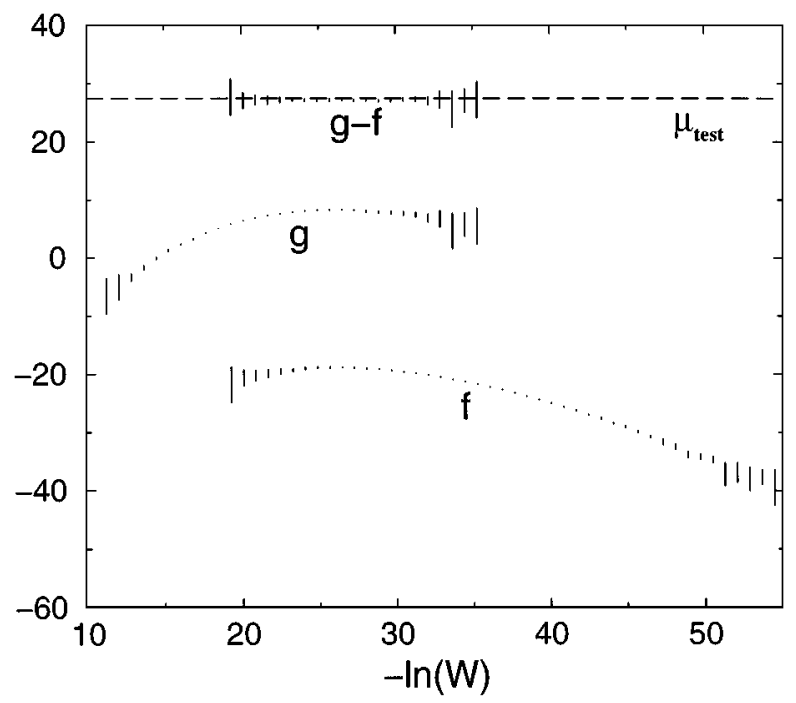

FIG. 4. Overlapping distribution for a chain of length 10 at $\rho=1.0$.

4.1.2. Overlapping distribution method. As explained above, the chemical potential of a chain can be calculated by adding this "ghost" chain into the simulation box. However, the same thing can be achieved by removing a real chain from the simulation box. The principle of the overlapping distribution method is to calculate a histogram of potential energy change when adding or removing the chain. If these two distributions overlap a reliable estimate of the chemical potential can be obtained. If these distributions do not overlap sampling problems may be expected [23].

The potential energy change of the chain addition is calculated with the method described in the previous section. The potential energy change of the real chain is calculated in a similar way. Again a number of trial orientations are chosen, but this time not $k$ but $k-1$. In addition to these $(k-1)$ trial orientations also the real orientation is used to calculate the Rosenbluth weight. For the real chain energy we have to perform a separate simulation for each chain length, while for the "ghost" chain method only a single simulation is required for the longest chain length, and store the results while growing the chain.

By constructing the following functions we can calculated the excess chemical potential ${ }^{1}$

$$
\begin{aligned}
& f(-\ln (W))=\ln \left(p_{0}(-\ln (W))\right)+\frac{1}{2} \ln (W) \\
& g(-\ln (W))=\ln \left(p_{1}(-\ln (W))\right)-\frac{1}{2} \ln (W) .
\end{aligned}
$$

In which $p_{0}$ is the probability density of $-\ln (W)$ in the case of adding a chain and $p_{1}$ of removing one. The chemical potential is found by subtracting these two functions,

$$
\beta \mu_{\text {test }}=g(-\ln (W))-f(-\ln (W)) .
$$

In Fig. 4 the functions $f, g$ and $g-f$ are shown as well as the chemical potential as calculated with the Rosenbluth test particle insertion method $\left(\mu_{\text {test }}\right)$, Eq. (11). As can be

\footnotetext{
${ }^{1}$ In the article of Mooij and Frenkel [23] the functions $f$ and $g$ contain some typographic errors and should read as Eq. (12).
} 


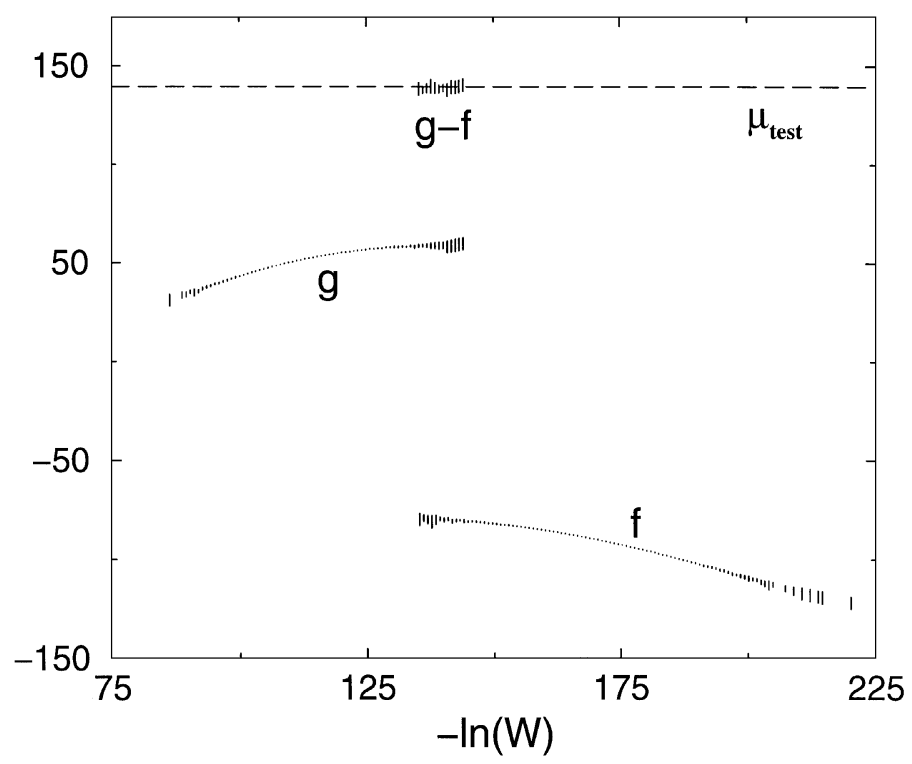

FIG. 5. Overlapping distribution for a chain of length 50 at $\rho=1.0$.

seen the overlap between the two is good for a chain with 10 beads. For a longer chain ( $l=50$; see Fig. 5 ) the overlap is smaller, only the tails of the distributions are overlapping. The error in these tails is larger then for $l=10$, so there is also a larger error in the estimation of the excess chemical potential. But these results indicate that even for $l=50$ the original Rosenbluth scheme still gives a reasonable result.

It is instructive to compare these results with the calculation of the chemical potential of a chain of Lennard-Jones beads in a solvent of Lennard-Jones particles [17]. For these Lennard-Jones chains a reliable chemical potential could be obtained for chain lengths up to 10 or 20 , depending on the density of the solvent. Here we could obtain a result for the chemical potential for much larger chains at acceptable densities. This is because of the relative softness of the potential.

\section{CONCLUDING REMARKS}

In this paper a combination of DPD and Monte Carlo techniques has been explored. We have shown that in the case of phase equilibria of a liquid-liquid mixture this combination gives a much more efficient sampling scheme. In addition, with this combined method one can obtain data on the coexistence properties much closer to the critical point. Another example concerns the calculation of the free energy of a polymer in solution. We have shown that at acceptable densities the excess chemical potential can be calculated for relatively long chains.

\section{APPENDIX}

In this Appendix simulation details are given for the DPD simulations, the Gibbs ensemble simulations, and the free energy calculations. 
TABLE I

The DPD Model Parameters

\begin{tabular}{cccccc}
\hline Parameter & $a_{i j}(i \neq j)$ & $a_{i j}(i=j)$ & $r_{c}$ & $\gamma$ & $\delta t$ \\
\hline Value & 30.0 & 25.0 & 1.0 & 3.0 & 0.06 \\
\hline
\end{tabular}

\section{DPD Simulations}

The DPD simulations were performed with parameters used by Groot and Warren [9]. They are displayed in Table I. The total number of particles was 6000 and the box size $20 \times 10 \times 10$. This rectangular shape was chosen to stabilize a planar interface. To obtain a density profile in the simulation box, 15000 time steps were needed to form the interface. Then the density was computed every 50 steps over 50000 time steps.

\section{Gibbs Ensemble Simulations}

The Gibbs ensemble simulations were performed with the potential that gives rise to the conservative force in the DPD scheme. A general description of this technique can be found in $[8,15]$. The simulations were performed in cycles. In every cycle, we select one of the following trial moves at random:

1. Displacement. A randomly chosen particle is given a random displacement. The maximum displacement is chosen such that $50 \%$ of the displacements are accepted.

2. Particle exchange. A component is selected at random. It is selected at random to transfer a particle of this component from box A to box B or vice versa. Finally, a particle in the correct box and of the correct component is chosen and transfered to a random position in the other box.

3. Identity change. At random, it is chosen to exchange a particle of component 1 in box A with a particle of component 2 in box B or vice versa. The acceptance/rejection rule of this trial move can be found in [24].

No change of volume was attempted because the mixture is symmetric. The number of trial moves in one cycle is equal to the number of particles. The moves were selected in such a way that on average $50 \%$ displacements, $40 \%$ exchanges, and $10 \%$ identity switches were performed. To equilibrate the two boxes in the simulation 500 cycles were used. Then the density of the two boxes was computed every 100 cycles for 20000 steps.

\section{Free Energy Calculations}

For the free energy calculations the parameters used by Groot and Warren [9] were chosen (see Table I, and $a_{i i}=25.0$ ). The box size for these simulations was $10 \times 10 \times 10$ and the number of particles was varied between 500 and 1000. To equilibrate the system 1000 time steps were used. The number of trial orientations was 10. The Rosenbluth factor was computed every time step over $5 \times 10^{4}$ time steps. The error was calculated using the block average method.

The overlap distributions were computed every 10 time steps over $2 \times 10^{4}$ time steps. To calculate the error in the distribution 10 distributions were calculated, leading to a total simulations time of $2 \times 10^{5}$ time steps. 


\section{ACKNOWLEDGMENTS}

Financial support provided to SMW by DSM Research, to TJHV from SON (Stichting Scheikundig Onderzoek Nederland), and to BS via the Graduate School on Process Technology (OSPT) is gratefully acknowledged. Part of the computer resources were generously provided by SARA (Stichting Academisch Rekencentrum Amsterdam). SMW thanks Rob Groot and Patrick Warren (Unilever Research, Port Sunlight) for introducing him to DPD simulations.

\section{REFERENCES}

1. P. J. Hoogerbrugge and J. M. V. A. Koelman, Simulating microscopic hydrodynamic phenomena with dissipative particle dynamics, Europhys. Lett. 19(3), 155 (1992).

2. P. Español, Hydrodynamics from dissipative particle dynamics, Phys. Rev. E 52(2), 1734 (1995).

3. P. Español and P. Warren, Statistical mechanics of dissipative particle dynamics, Europhys. Lett. 30(4), 191 (1995).

4. I. Pagonabarraga, M. H. J. Hagen, and D. Frenkel, Self-consistent dissipative particle dynamics, Europhys. Lett. 42(4), 377 (1998).

5. J. M. V. A. Koelman and P. J. Hoogerbrugge, Dynamic simulation of hard-sphere suspensions under steady shear, Europhys. Lett. 21(3), 363 (1993).

6. A. G. Schlijper, P. J. Hoogerbrugge, and C. W. Manke, Computer simulation of dilute polymer solutions with the dissipative particle dynamics method, J. Rheo. 39(3), 567 (1995).

7. K. E. Novik and P. V. Coveney, Using dissipative particle dynamics to model binary immiscible fluids, Int. J. Mod. Phys. C 8(4), 909 (1997).

8. A. Z. Panagiotopoulos, Direct determination of phase coexistence properties of fluids by Monte Carlo simulation in a new ensemble, Mol. Phys. 61, 813 (1987).

9. R. D. Groot and P. B. Warren, Dissipative particle dynamics: Bridging the gap between atomistic and mesoscopic simulation, J. Chem. Phys. 107(11), 4423 (1997).

10. B. Smit, Ph. de Smedt, and D. Frenkel, Computer simulations in the Gibbs ensemble, Mol. Phys. 68, 931 (1989).

11. J. R. Recht and A. Z. Panagiotopoulos, Finite-size effects and approach to criticality in Gibbs ensemble simulations, Mol. Phys. 80, 843 (1993).

12. A. Z. Panagiotopoulos, Molecular simulations of phase coexistence: Finite-size effects and the determination of critical parameters for two- and three-dimensional Lennard-Jones fluids, Int. J. Thermophys. 15, 1057 (1994).

13. K. K. Mon and K. Binder, Finite size effects for the simulation of phase coexistence in the Gibbs ensemble near the critical point, J. Chem. Phys. 96, 6989 (1992).

14. B. Widom, Some topics in the theory of fluids, J. Chem. Phys. 39, 2802 (1963).

15. D. Frenkel and B. Smit, Understanding Molecular Simulations: From Algorithms to Applications (Academic Press, San Diego, 1996).

16. J. Harris and S. A. Rice, A lattice model of a supported monolayer of amphiphilic molecules: Monte Carlo simulations, J. Chem. Phys. 88, 1298 (1988).

17. D. Frenkel, G. C. A. M. Mooij, and B. Smit, Novel scheme to study structural and thermal properties of continuously deformable molecules, J. Phys.: Condens. Matter 4, 3053 (1992).

18. J. J. de Pablo, M. Laso, and U. W. Suter, Simulation of polyethylene above and below the melting point, J. Chem. Phys. 96, 2395 (1992).

19. J. I. Siepmann and D. Frenkel, Configurational-bias Monte Carlo: A new sampling scheme for flexible chains, Mol. Phys. 75, 59 (1992).

20. M. N. Rosenbluth and A. W. Rosenbluth, Monte Carlo simulations of the average extension of molecular chains, J. Chem. Phys. 23, 356 (1955).

21. H. Flyvbjerg and H. G. Petersen, Error estimates on averages of correlated data, J. Chem. Phys. 91, 461 (1989). 
22. C. H. Bennett, Efficient estimation of free energy differences from Monte Carlo data, J. Comput. Phys. 22, 245 (1976).

23. G. C. A. M. Mooij and D. Frenkel, The overlapping distribution method to compute chemical potentials of chain molecules, J. Phys.: Condens. Matter 6, 3879 (1994).

24. A. Z. Panagiotopoulos, N. Quirke, M. Stapleton, and D. J. Tildesley, Phase equilibria by simulations in the Gibbs ensemble: Alternative derivation, generalization and application to mixtures and membrane equilibria, Mol. Phys. 63, 527 (1988). 